\title{
ST-segment deviations during pacing-induced increased heart rate in patients without coronary artery disease
}

\author{
Sören Häggmark ${ }^{1}$, Michael F. Haney ${ }^{2}$, Steen M. Jensen ${ }^{3}$, Göran Johansson ${ }^{2}$ and Ulf Näslund ${ }^{3}$ \\ ${ }^{1}$ Cardiothoracic Surgery, Heart Centre, University Hospital, Umeå, ${ }^{2}$ Department of Surgical and Perioperative Science, Anaesthesiology and Intensive Care Medicine, \\ Umeå University, and ${ }^{3}$ Cardiology, Heart Centre, University Hospital and Department of Public Health and Clinical Medicine, Umeå University, Umeå, Sweden
}

\section{Summary}

\section{Correspondence \\ Sören Häggmark, Heart Centre, University Hospital, Umeå, SE-901 85 Umeå, Sweden \\ E-mail: soren.haggmark@vll.se}

\section{Accepted for publication}

Received 4 January 2005;

accepted 19 April 2005

\section{Key words}

electrocardiography; heart rate; myocardial ischemia; ST segment; tachycardia; vectorcardiography
Introduction: In order to interpret ST-segment changes as an indicator of ischemia in patients with higher heart rates (HRs), the relation between ST-segment levels and HR needs to be well defined in subjects without coronary artery disease.

Methods: Eighteen patients with normal ECGs in the catheterization laboratory, after radiofrequency ablation of AV nodal re-entry tachycardia or an accessory pathway were included. Computerized online vectorcardiography (VCG) was performed during step-wise atrial pacing-induced increases in HR up to 150 beats $\mathrm{min}^{-1}$ (bpm). The ST-vector magnitude (ST-VM) and the relative ST change vector magnitude (STC-VM) were analysed at the J point, J +20 and J $+60 \mathrm{~ms}$.

Results: There was no divergence in the course of ST-VM or STC-VM based on J point +0 , 20, or $60 \mathrm{~ms}$ during increasing HR. The STC-VM mean values increased progressively during increases in HR above $100 \mathrm{bpm}$, with an average increase in STC-VM of 15-20 $\mu \mathrm{V}$ per $10 \mathrm{bpm}$ increases in HR. The ST-VM response during HR increases showed a heterogeneous and unpredictable pattern.

Conclusion: The STC-VM increases linearly with rising HRs above $100 \mathrm{bpm}$. The STCVM can exceed widely recognized ischemic thresholds during higher HRs in the absence of ischemia. The choice of J point time to ST-VM measurements as tested here is not important for the STC-VM relation to HR at these HR levels. Further clinical testing is needed to improve the diagnostic specificity of STC-VM measurements during increased HRs.

\section{Introduction}

Several conditions other than ischemia, such as increased heart rate (HR) during physical or emotional stress, may cause marked changes in ST levels. Adjustments of STdeviations for increases in HR during exercise ECG were proposed already 25 years ago (Elamin et al., 1982) in order to enhance the discrimination between myocardial ischemia and physiological ST changes during conditions of increased HR. Despite this, there is no consensus concerning the diagnostic and predictive value of adjustments of ST-segment changes for increases in HR.

There are no previous studies using vectorcardiography (VCG), which have been specifically designed to analyse the impact of HR changes on the VCG-ST parameters. However, a relationship between VCG deviations and HR has been suggested based on observations in observations from two exercise levels in patients with ischemic heart disease (Pilhall et al., 1992) and for pacing controlled HRs at two levels (Jensen et al., 1997), but the type of relation and its nature has not yet been well characterized. Criteria for detection of ischemia with VCG have been empirically established (Dellborg et al., 1991; Näslund et al., 1993; Jensen et al., 1997) without reference to HR.

Another problem in ST-segment analysis for detection of ischemia concerns where to measure the ST-deviation in temporal relation to the $\mathrm{J}$ point. In the recent ACC/ESC consensus statement for the definition of acute myocardial infarction (Alpert et al., 2000) it was recommended (without any references) to calculate the ST deviation at the $\mathrm{J}$ point. During exercise ECG, the ST deviation is most frequently measured at $\mathrm{J}+60$ or $\mathrm{J}+80 \mathrm{~ms}$ (Wilson \& Johnston, 1938). In most modern ECG equipment, the point for automatic assessment can be manually adjusted in the software from between 0 to $80 \mathrm{~ms}$ or more after the J point. However, the $\mathrm{J}$ point is not always easily defined by, manual or automatic 
algorithms using vector acceleration. Furthermore, during tachycardia with HRs reaching 140-160, the $\mathrm{T}$ wave begins in close proximity to $\mathrm{J}+60-80 \mathrm{~ms}$, which may derange the measurement of the ST level. In clinical practice, the importance of these problems is most likely underestimated.

We hypothesized that HR increases per se cause ST vector changes in the absence of ischemia, and that a systematic relation between HR increases or absolute HR levels and ST changes in the absence of ischemia can be described in subjects without coronary artery disease. We further hypothesized that the analyses of ST segment at $\mathrm{J}+0, \mathrm{~J}+20$, or $\mathrm{J}+60 \mathrm{~ms}$ will result in different levels of ST magnitude change. We conducted a study to test these hypotheses using computerized VCG during atrial pacing-related increases in HR.

\section{Methods}

\section{Patients}

This study was conducted with the approval of the Human Research Ethics Committee of Umeå University, and in accordance with the Helsinki Declaration (2000) for Ethical Principles for Medical Research Involving Human Subjects. Patients 20-80 years of age, scheduled for ablation therapy for atrioventricular (AV) nodal re-entrant tachycardia, WolfParkinson-White syndrome with concealed accessory pathway or ectopic atrial tachycardia, were considered for participation in the study. A medical history not suggestive of coronary artery disease, a normal ECG and absence of hypertrophy by echocardiography was required for inclusion. In patients older than 55 years of age, a normal coronary angiogram was required. The patients were assessed by, ECG, echocardiography, and coronary angiography (for patients over age 55).

The patients were treated with radiofrequency ablation therapy in the electrophysiology laboratory. Enrolment in the study was made the day before the ablation procedure after obtaining informed consent. Participation in the study occurred only if the ablation procedure was completed successfully. Patients were free to decline participation at any time. All chronic medications were discontinued 4 days before investigation, and no medication was given before or during the procedure (no atropine was given).

\section{Catheterization}

After the ablation procedure a $5 \mathrm{~F} 4$ electrode catheter was placed in a high lateral position in the right atrium. In five patients a $7 \mathrm{~F}$ single lumen catheter (Inquiry Lume Seerable Curv, Ib Irvine Biomedical, CA, USA) was placed in the coronary sinus for blood sampling. In these five patients a radial arterial catheter was introduced for arterial blood sampling. Pacing was accomplished using a standard system for intra-cardiac bipolar pacing and recording (EP-Tracer Version 3.0, CardioTek, Maastricht, Netherlands).

\section{Pacing protocol}

Baseline pacing was started at a rate 5-10 bpm higher than the patient's resting HR in order to avoid interference from spontaneous beats. The baseline-pacing period lasted for $6 \mathrm{~min}$. The pacing rate was increased stepwise with $10 \mathrm{bpm}$ for periods of $4 \mathrm{~min}$ until the Wenchebach point occurred or the HR reached $150 \mathrm{bpm}$, whichever came first. Arterial and coronary sinus blood samples were drawn simultaneously for analysis of blood lactate (YSI 2300 STAT plus, YSI Inc., Yellow Springs, OH, USA) after $3.5 \mathrm{~min}$ into each pacing period (for five patients). Systolic and diastolic blood pressures were measured for each period (Marquette SOLAR 9000).

\section{Measurements}

Blood pressure was measured non-invasively (Marquette SOLAR 9000) before the start of the protocol, and then at each HR level (pacing level). The HR was derived from the ECG R-R intervals (MIDA 1000, Ortivus Medical AB, Täby, Sweden).

\section{Computerized on-line vectorcardiography}

Eight VCG electrodes were placed according to the lead system described by Frank (1956). The MIDA 1000 VCG system with MIDA1000 2.74 software (Ortivus Medical AB, Täby, Sweden) was used for recording of the VCG. This system has been described previously in detail (Sederholm et al., 1983; Dellborg et al., 1991; Näslund et al., 1993). The signals were sampled at $500 \mathrm{~Hz}$ in three orthogonal components $\mathrm{X}, \mathrm{Y}$ and $\mathrm{Z}$. For averaged complexes, the measurement sensitivity was $1.0 \mu \mathrm{V}$.

\section{The ST deviation at J + o, J + 20 and J + $60 \mathrm{~ms}$}

During the baseline period, all complexes were recorded and averaged for periods of $2 \mathrm{~min}$. After the baseline period, periods of $15 \mathrm{~s}$ were used to generate an averaged heart cycle signal. For each 4-minute pacing period, the second and third minutes in each period were analysed. The VCG ST vector trend variables were derived from measured voltages in the X-, Y- and Z-leads automatically for ST at J +20 and $J+60 \mathrm{~ms}$. From this summary complex, the ST vector at $\mathrm{J}+0 \mathrm{~ms}$ was derived by reading the $\mathrm{X}, \mathrm{Y}$ and $\mathrm{Z}$ magnitudes at the J point, and then calculating the ST vector variables STVM and STC-VM.

The absolute ST-VM was calculated as follows:

$$
\mathrm{ST}-\mathrm{VM}=\sqrt{\mathrm{X}^{2}+\mathrm{Y}^{2}+\mathrm{Z}^{2}} \mu \mathrm{V} .
$$

The relative spatial difference between reference and current ST vector was calculated as follows.

$$
\mathrm{STC}-\mathrm{VM}=\sqrt{\left(\mathrm{X}_{\mathrm{i}}-\mathrm{X}_{0}\right)^{2}+\left(\mathrm{Y}_{\mathrm{i}}-\mathrm{Y}_{0}\right)^{2}+\left(\mathrm{Z}_{\mathrm{i}}-\mathrm{Z}_{0}\right)} \mu \mathrm{V} \text { where }
$$
the ' 0 ' subscript represents the baseline reference measurement, and the ' $i$ ' subscript represents the current measurement. 


\section{Calculations and statistics}

Relative lactate change in concentration for the coronary circulation was calculated as follows: ([lactate $\left.{ }_{\text {art }}\right]-$ [lactate $\left.\left._{\mathrm{CS}}\right]\right) /\left[\right.$ lactate $\left._{\text {art }}\right] \times 100$, expressed as a percent $(\mathrm{CS}=$ coronary sinus) (Häggmark et al., 1989). Body mass index (BMI) was calculated as mass height ${ }^{-2}\left(\mathrm{~kg} \mathrm{~m}^{-2}\right)$.

The ST-VM and STC-VM values were grouped for absolute HRs, with mean values \pm standard error of the mean $( \pm$ SEM) or with the confidence interval (CI). STC-VM values at baseline $\mathrm{HR}$ and baseline plus $10 \mathrm{bpm}$ levels were calculated as mean \pm 2 standard deviations (SD). All observations above 2 $\mathrm{SD}$ were included in a linear regression analysis to calculate in each individual the slope of STC-VM in relation to HR (STCVM/HR slope). In addition, the mean STC-VM/HR slope for all individuals at each HR (ST/HR index) was calculated. Analyses were performed to detect differences in ST-VM and STC-VM for $\mathrm{J}+0, \mathrm{~J}+20$, and $\mathrm{J}+60 \mathrm{~ms}$ using ANOVA and the paired t-test. A P $<0.05$ was used to determine statistical significance.

\section{Results}

One patient was excluded from analysis due to technical problems with the atrial pacing. Results for the remaining eighteen patients, eight males and 10 females, were analysed. The mean age was $44 \cdot 3 \pm 9 \cdot 3$ years (SD), with range 32-64. Mean BMI was $25 \cdot 0 \pm 4 \cdot 3 \mathrm{~kg} \mathrm{~m}^{-2}(\mathrm{SD})$, range $18 \cdot 3-37 \cdot 1 \mathrm{~kg} \mathrm{~m}^{-2}$. Pacinginduced increases in HR were achieved for all 18 patients for 10, 20 and $30 \mathrm{bpm}$ over baseline. Sixteen patients were paced to 40 bpm over baseline, with 12 and 6 patients reaching 50 and $60 \mathrm{bpm}$ over baseline. No statistical analysis was performed for the six measurements at $60 \mathrm{bpm}$ over the baseline pacing level. Mean blood pressure at baseline pacing HR was $93.5 \pm 1.8 \mathrm{~mm}$ $\mathrm{Hg}$, and then for successive pacing levels $97 \cdot 0 \pm 2 \cdot 0,99 \cdot 2 \pm 2 \cdot 0$, $100 \cdot 4 \pm 1 \cdot 9,101 \cdot 3 \pm 2 \cdot 2$ and $101 \cdot 3 \pm 3 \cdot 4 \mathrm{~mm} \mathrm{Hg}$ (SEM). Coronary sinus lactate values were collected for five patients. Relative mean lactate concentrations in the coronary circulation during HR increases ranged from $15 \cdot 6$ to $25 \cdot 3 \%$ with mean $17 \cdot 0 \pm 5 \cdot 7 \%$ (SEM), with all observations demonstrating net lactate consumption (no net production).

\section{The ST-VM and STC-VM at J, J + 20 and J + $60 \mathrm{~ms}$}

ST-segment shape or morphology demonstrated no horizontal or down-sloping ST changes. Mean ST-VM measured at $\mathrm{J}+60 \mathrm{~ms}$ was higher than at $\mathrm{J}$ point 0 or $\mathrm{J}+20 \mathrm{~ms}$ up to HRs of approximately $120 \mathrm{bpm}$, but there were wide CIs (Fig. 1, Panel a). No apparent difference was found between the mean trends over the whole course of ST-VM from baseline pacing to the end of pacing. The values for ST-VM measured at different time intervals from J, as HR increased, did not diverge. Paired comparisons for ST-VM with $\mathrm{J}+20$ and $\mathrm{J}+60 \mathrm{~ms}$ showed a higher absolute ST-VM up to HR $120 \mathrm{bpm}$, while there was no difference between $\mathrm{J}+20$ and $\mathrm{J}+60 \mathrm{~ms}$ for the highest HRs. Paired comparisons of $\mathrm{J}+20$ and $\mathrm{J}+0$ (also (a)

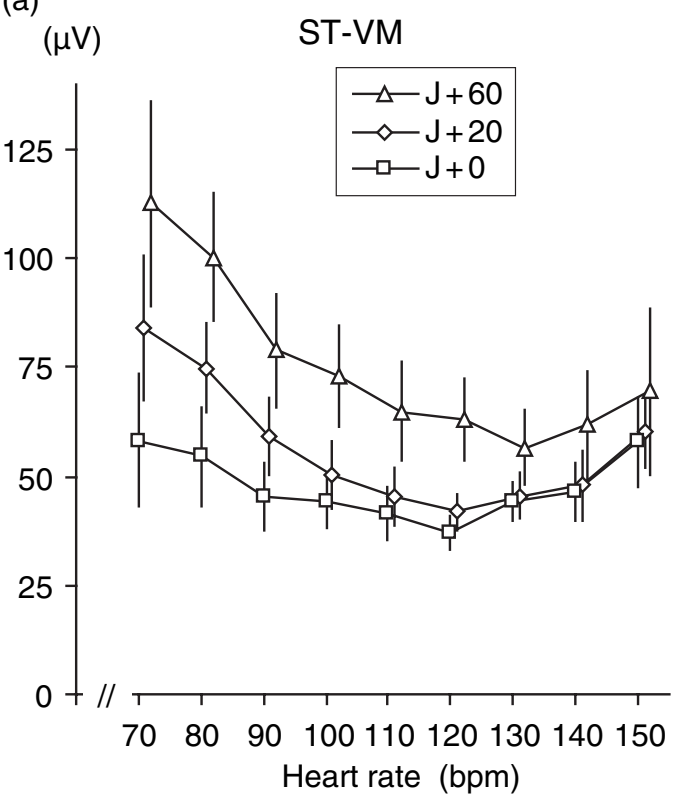

(b)

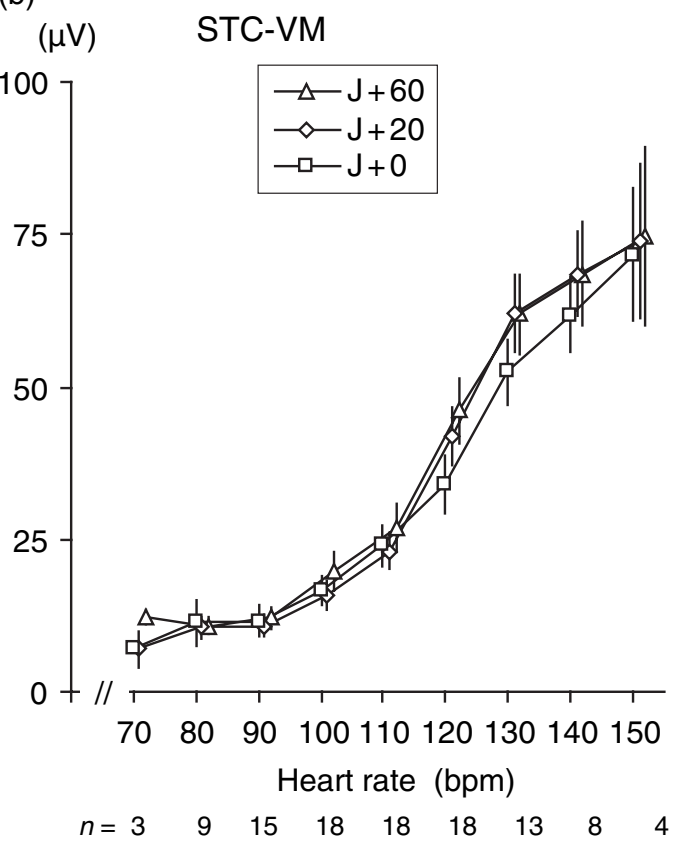

Figure 1 Panel (a) shows ST-VM calculated at $J+0, J+20$ and $\mathrm{J}+60 \mathrm{~ms}$ (mean \pm SEM), respectively at paced HRs from 70 to $150 \mathrm{bpm}$. Note that even at high HRs, there is no apparent increase in ST-VM, including for ST-VM J $+60 \mathrm{~ms}$, which might have been expected if the ST measurement was disturbed by T wave interference. Panel (b) shows STC-VM for the same measurements. There is obvious agreement for STC-VM measurements for the different temporal relations to the $\mathrm{J}$ point, over the whole range of HRs (Individual values are shown for $J+20$ in Fig. 2 and 4 )

$\mathrm{J}+20$ and $\mathrm{J}+60$ ) for STC-VM showed no difference at any HR (see Fig. 1, Panel b). In subsequent analyses measurements at $\mathrm{J}+20 \mathrm{~ms}$ were used. 


\section{ST-VM in relation to increased HR}

Individual data for ST-VM in relation to HR for all 18 patients is demonstrated in Fig. 2. Note that at HR 70 there are only three subjects, and only four subjects at HR $150 \mathrm{bpm}$. The individual variations in ST-VM levels at baseline were large. No systematic or predictable pattern for ST-VM during increased HR could be demonstrated. The mean ST-VM for each HR is shown in Fig. 2, which made subsequent in-depth analysis not meaningful.

The $\mathrm{X}, \mathrm{Y}$ and $\mathrm{Z}$ orthogonal complexes were examined qualitatively for each individual for ST-segment morphology during HR increases. There were ST-VM and STC-VM changes during HR increases in all patients, but there was no change in $\mathrm{X}$, Y or Z ST-segment morphology (no horizontal or downsloping ST segments).

A representative example of the ST-segment in the orthogonal leads X, Y and Z with HR (Fig. 3, Panel a), and ST-VM with STC-VM (Fig. 3, Panel b) demonstrates how, in 13/18 subjects, the resulting ST-VM is influenced by the proximity to zero (see the Pythagorean formula for calculation of ST-VM in the Methods section) as well as the direction of orthogonal lead voltage change. The STC-VM, however, indicates the systematic change in $\mathrm{X}, \mathrm{Y}$ and $\mathrm{Z}$ during $\mathrm{HR}$ increases.

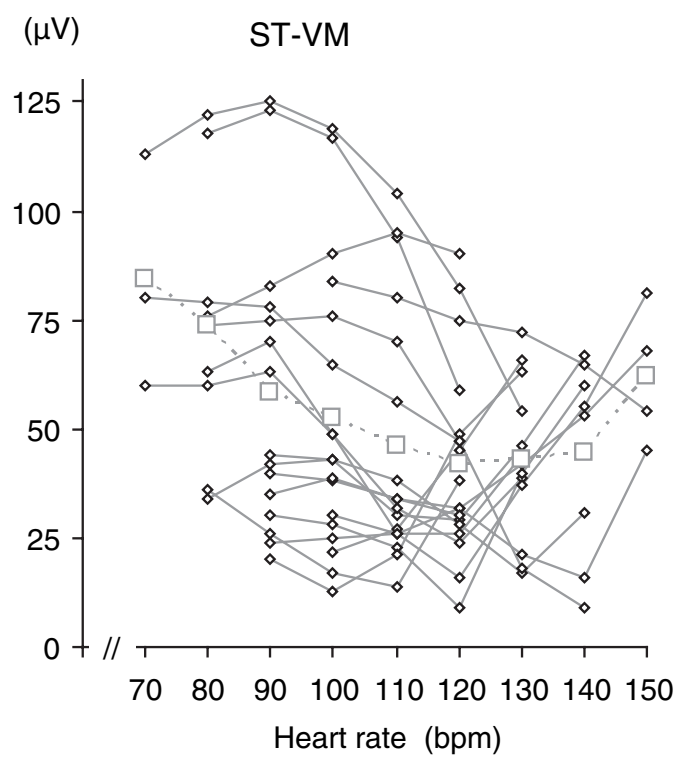

Figure 2 The ST-VM calculated at J $+20 \mathrm{~ms}$ for all subjects is shown during pacing-induced HR increases. Mean values at each HR level are shown, though these clearly do not reflect the individual starting magnitudes or course.

\section{STC-VM in relation to increased HR}

Individual data and mean values for STC-VM at absolute HRs are shown in Fig. 4. The STC-VM at baseline pacing was approximately $10 \mu \mathrm{V}$, which reflected the amount of variability in STC$\mathrm{VM}$ between the reference at the start of the recording and the first pacing step. Increases in STC-VM above 2 SD occurred at approximately $110 \mathrm{bpm}$ as outlined in Fig. 4. Mean STC-VM
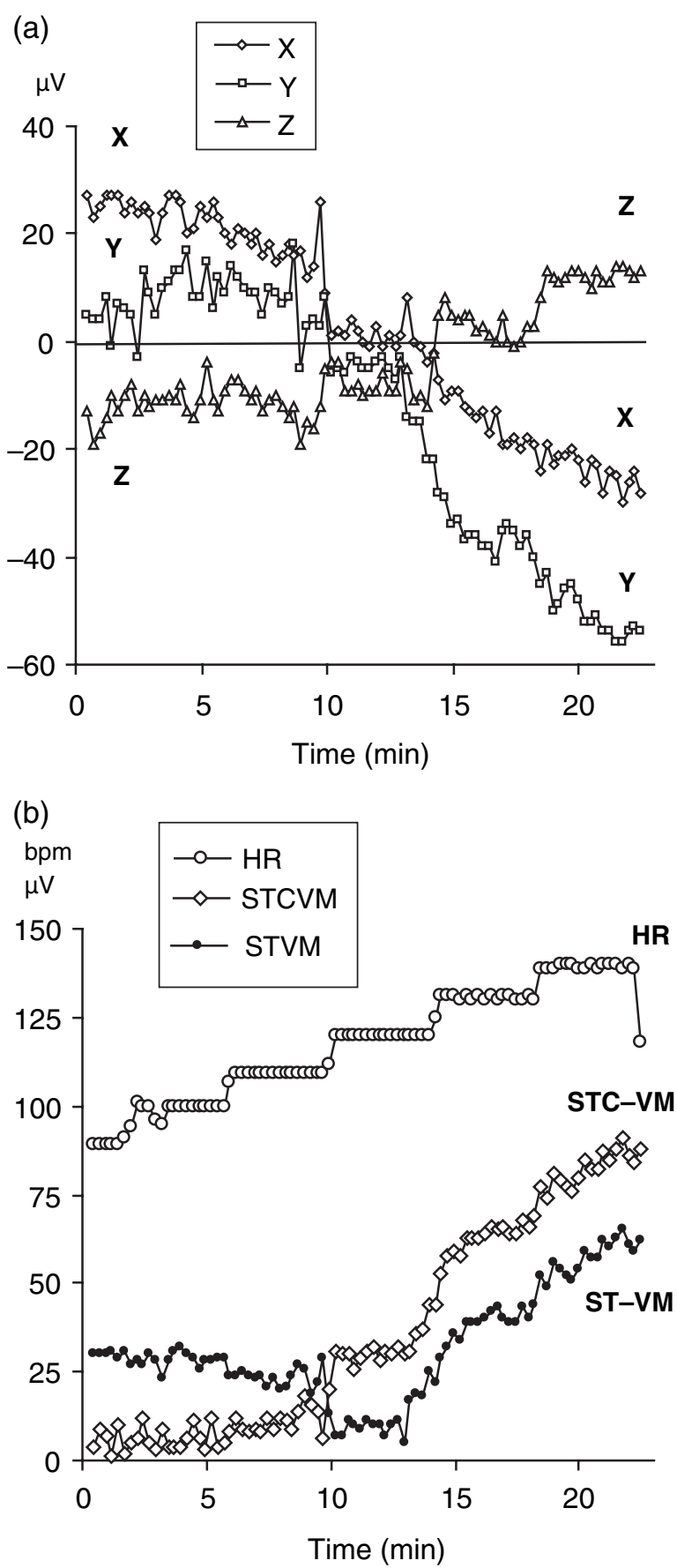

Figure 3 Panel (a) is a representative example of the ST magnitude in the orthogonal leads X, Y and Z, where ST-segment values during the course of pacing approach and cross zero. Panel (b) outlines paced HR, ST-VM, and STC-VM for the same subject. The ST-VM course changes direction when the orthogonal values cross zero, while STC-VM more accurately reflects change in magnitude and direction.

values for HRs above $100 \mathrm{bpm}$ examined in a regression analysis demonstrated a linear relationship with increased STC$\mathrm{VM}$ in relation to $\mathrm{HR}$ of $15.3 \mu \mathrm{V}$ per $10 \mathrm{bpm}$ (mean 'ST/HR index') increased HR over the range of HRs 110, 120, 130 and 140 bpm (Fig. 4). Observations at 150 bpm were not included 


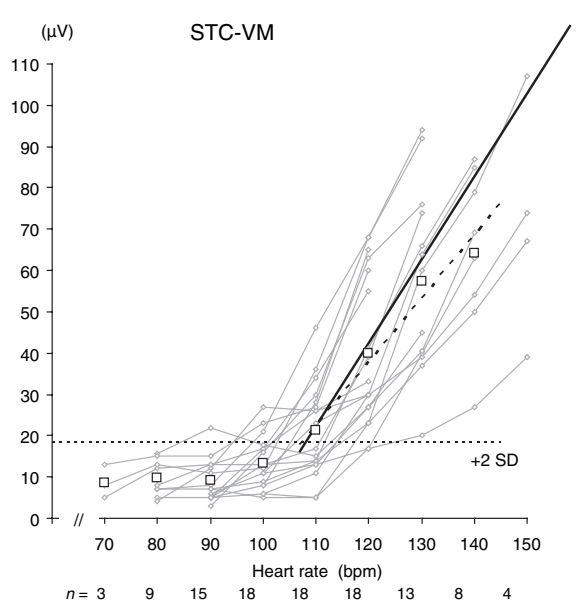

\begin{tabular}{rr}
\hline Pat. no & Slope \\
\hline 1 & $3 \cdot 30$ \\
2 & $2 \cdot 85$ \\
3 & $1 \cdot 26$ \\
4 & $0 \cdot 73$ \\
5 & $1 \cdot 45$ \\
6 & $0 \cdot 70$ \\
7 & $2 \cdot 44$ \\
8 & $2 \cdot 41$ \\
9 & $2 \cdot 71$ \\
10 & $2 \cdot 30$ \\
11 & $2 \cdot 75$ \\
12 & $0 \cdot 40$ \\
13 & $2 \cdot 10$ \\
14 & $1 \cdot 60$ \\
15 & $2 \cdot 30$ \\
16 & $2 \cdot 10$ \\
17 & $1 \cdot 85$ \\
18 & $2 \cdot 80$ \\
\hline Mean & $2 \cdot 00$ \\
SD & $0 \cdot 83$ \\
SEM & $0 \cdot 19$
\end{tabular}

Figure 4 Individual and mean values for STC-VM at J $+20 \mathrm{~ms}$ are shown in relation to paced HRs. The horizontal dotted line is $2 \mathrm{SD}$ from the mean STC-VM values at baseline and baseline $+10 \mathrm{bpm}$, as described in the text. The regression equation for the mean STC-VM values at HRs 110, 120, 130 and $140 \mathrm{bpm}$ is as follows: $y=1.53 \mathrm{x}-$ $146(\mathrm{r}=0.67, \mathrm{P}<0.001)$, which is shown as the hatched line. The unbroken line represents the mean slope of all individual slopes calculated for all observations of STC-VM which were above the 2 SD limit. To the right of the graph, individual values of the STC-VM/HR slope are shown.

in the calculation, since there were only four individuals reaching $150 \mathrm{bpm}$ and these subjects would have had an unreasonable weight in the mean STC-VM values. The mean STC-VM/HR slope calculated from the slope of all individuals was $2 \cdot 00 \pm 0.19 \mu \mathrm{V}$, corresponding to $20 \mu \mathrm{V}$ per $10 \mathrm{bpm}$ increase (Fig. 4). Fourteen of eighteen patients achieved a STCVM over $50 \mu \mathrm{V}$ during pacing, with the average STC-VM of $57 \cdot 3 \pm 6 \cdot 3 \mu \mathrm{V}$ at $\mathrm{HR} 130 \mathrm{bpm}$, range $34-98 \mu \mathrm{V}$. In one subject, STC-VM increased to $107 \mu \mathrm{V}$ at $150 \mathrm{bpm}$.

\section{Discussion}

The main findings in this study were that STC-VM increases were consistently demonstrated in relation to step-wise HR increases in clinical observations where the possibility for ischemia during HR changes was minimized. The STC-VM trends were relatively flat at lower HRs and steep at HRs above $100 \mathrm{bpm}$. The STC-VM in these two HR ranges were interpreted as both being linear, and therefore confirmed the hypothesis that a well-refined HR adjustment might be identified for STchanges. Expressed as a ST/HR index or ST/HR, slope increases for HRs in the range of 100-140 bpm were approximately 15$20 \mu \mathrm{V}$ per $10 \mathrm{bpm}$ increased HR in these subjects, with no signs of ischemia or coronary artery disease. Another novel finding in this experiment is that pacing-induced HR increases, with HRs common for coronary care monitoring, consistently produced STC-VM signals in ranges widely recognized to indicate ischemia. An increase in STC-VM or ST-VM of more than $50 \mu \mathrm{V}$ has been used as a threshold for myocardial ischemia in previous studies using computerized VCG (Dellborg et al., 1991;
Näslund et al., 1993; Jensen et al., 1994, 1997, 2002). Furthermore, ST-VM variability allowed no possibility to detect a relationship of ST-VM changes and HR changes. This is not able since ST-VM is so commonly implemented in detection and monitoring of myocardial ischemia in patient care settings. During increased HR, ST-VM may increase, remain unchanged, or even decrease.

\section{ST-segment magnitude relationships to HR}

Analysis of these results suggested a biphasic linear relationship for HR and STC-VM increases. The STC-VM did not change at lower HRs, but increased progressively in the higher HR range. One clinical implication for the lower HR range, at $100 \mathrm{bpm}$ or below, is that STC-VM requires no adjustment with reference to $\mathrm{HR}$ in that range. Observations of increased STC-VM levels at higher HRs provide motivation to improve specificity for ischemia in ST-segment analysis either through a correction for HR or further consideration for an ischemic threshold level. In this study material, STC-VM values above $50 \mu \mathrm{V}$ occurred in one third of these patients when HRs were 120 , which is a HR quickly achieved during exercise testing, ischemia, or heart failure. While the STC-VM observations at higher HRs appeared to be highly linear, prospective investigations of a correction factor for HR (e.g. using ST/HR index or ST/HR slope) are needed before clinical introduction of a formal correction algorithm.

Previous studies (Lehtinen et al., 1996; Bjurö et al., 2004) have suggested patterns for ST changes during increased HR in exercise testing, and our results correspond well with several 12 lead ECG studies to describe such algorithms, including an ST/ HR index (Kligfield et al., 1989; Lachterman et al., 1990) or ST/ HR slope (Okin \& Kligfield, 1989) for ST magnitude adjustment or for improved specificity for ischemia criteria. The published experience with vectorcardiographic ST magnitudes in relation to HR and QRS magnitude demonstrated an elevated STC-VM at maximal exercise in healthy subjects but did not explore the relation of stepwise HR increases and STC-VM (Pilhall et al., 1992). These studies are all limited in that there was no second method to identify or rule out ischemia.

There are several possible well-recognized mechanisms by which ST-segment magnitude changes can occur during HR increases. Body habitus, body position, and even gender may need to be considered when interpreting STC-VM changes, though a much larger study material would be needed to identify independent effects of these factors on STC-VM response to HR increases. Vector mathematical factors (Fig. 3) or encroachment of the $\mathrm{T}$ wave into ST-segment measurements at higher HRs, were not a factor in these findings. This study, however, was not designed to elucidate the mechanism for these STC-VM increases.

Concerning the clinical specificity of ST-VM for detection of ischemia, a striking finding was that ST-VM demonstrated variability such that there was no possibility to detect a specific relationship between ST-VM changes and HR changes. The ST- 
VM values during increasing HR were highly variable: some started at higher magnitudes, some started at lower, some increased and some decreased. We were not able to find any pattern in ST-VM response during increasing (paced) HR. These findings lead to concern for reliability in clinical applications of ST-VM for detection of ischemia during conditions with significant variations in HR. ST-VM is the most widely used vectorcardiographic parameter in ischemia monitoring in coronary care units. We believe that the influence of HR changes on ST-vector changes is frequently not adequately taken into account, particularly when interpreting ST changes in trend curves. There is a risk that ST-VM or STC-VM events during HR increases can easily be interpreted as caused by myocardial ischemia, when they may actually be due to other causes.

\section{ST-segment measurement point}

STC-VM changes in this material were not affected by, different temporal relations to the J point. Current guidelines for STsegment measurements do not specify a point in the ST-segment at which the ST-deviation should be measured (Okin et al., 1991; Gibbons et al., 2002). The findings in the present study also disprove the widespread concern that $\mathrm{J}$ point plus $60 \mathrm{~ms}$ leads to ST measurement disturbances related to the $\mathrm{T}$ wave in ranges of HR increase up to $150 \mathrm{bpm}$ (Savvides et al., 1983). The J point offset to ST-segment measurement is one of many aspects of ST-segment analysis, which can influence the assessment of ischemia. There is concern that measurement of ST-level at $J+0$ is very sensitive to change if the J point lies on a steep sloping signal. Theoretically, a small difference in time can lead to a relatively large difference in ST magnitude. The STsegment at $\mathrm{J}+20$ or $\mathrm{J}+60 \mathrm{~ms}$ is more likely to reside on a plateau, and small differences in time from $J$ are less likely to result in large $\mu \mathrm{V}$ differences. Therefore, $\mathrm{J}+0$ observations are probably more prone to artifactual disturbances in measurement artefact than for observations at J +20 and $\mathrm{J}+60 \mathrm{~ms}$. One possible interpretation is that several different $J$ point offsets can be used, up to HRs of $150 \mathrm{bpm}$, without concern for $\mathrm{T}$ wave encroachment on the ST-segment measurement. J point offset with $80 \mathrm{~ms}$ was not examined here. The lack of consensus for J point offset for ST-segment measurement for detection of ischemia is bothersome during clinical ST-segment analysis, and further study and discussion for professional guidelines are needed. From the findings in this study, there is no evidence to support or rule out one or another J point offset.

\section{Other electrophysiological influences on ST magnitude}

Atrial repolarization $\left(\mathrm{T}_{\mathrm{a}}\right)$ has a duration, which can coincide with both the QRS complex, the J point, and even early portions of the ST-segment (Hayashi et al., 1976; Mirvis \& Wilson, 1986; Debbas et al., 1999). The later phases of the $T_{a}$ wave generate very low voltages by surface electrocardiography. The $\mathrm{T}_{\mathrm{a}}$ wave at its height (occurring shortly before or during the start of the QRS complex) can provide an ST- segment change of up to $50 \mu \mathrm{V}$, though a rough estimate of $\mathrm{T}_{\mathrm{a}}$ wave voltage of not higher than $10-15 \mu \mathrm{V}$ is reasonable for $T_{a}$ during the early part of the ST segment (if $T_{a}$ coincides at all with the ST). Also, since the P-Q interval increases physiologically with increasing HRs, the likelihood that $T_{a}$ coincides with the ST measurements decreases at higher HRs. We observed that ST-VM increased the farther in time from the $\mathrm{J}$ point that it was measured, and the higher the HR, the closer the voltages for ST-VM for ST-VM $\mathrm{V}_{\mathrm{J}+0}, \mathrm{ST}-\mathrm{VM}_{\mathrm{J}+20}$ and ST- $\mathrm{VM}_{\mathrm{J}+60}$ (see Fig. 1). This would support the interpretation that there is a possible net influence from $T_{a}$ present at $J$ at lower HRs, which decreased progressively (or resolved) from $\mathrm{J}+0$ to $\mathrm{J}+20$ and $\mathrm{J}+60 \mathrm{~ms}$ as well as during increased HRs. Still, this evidence is indirect.

\section{Limitations}

There were several limitations in the study design. First, ischemia cannot be definitively ruled out for all patients, though careful patient selection and no ischemia as judged by coronary lactate analyses in a small subset of five subjects allow a presumption that ischemia was not present during the data collection. Practical study considerations limited the number of subjects in whom coronary sinus lactate (transcoronary lactate differences) could be measured. Lactate sampling from the coronary sinus has limitations for detecting regional ischemia, since lactate is both locally consumed and produced in cardiac myocytes, and coronary sinus sampling is not heart regionspecific.

Second, pacing-induced HR increases may not mirror exactly the conditions where high HRs occurs during clinical ischemia detection in patients at high risk. Clinical ischemia is produced by conditions, which involve limitations in coronary blood flow often together with increase in myocardial work. Factors other than simple HR may be involved in or mitigate STC-VM events during high HRs brought about by increased myocardial workload.

Third, there were relatively few observations at the lowest (70 bpm) and highest (150 bpm) HRs. The experimental protocol succeeded in providing conditions or HRs, which are common in range to those seen during exercise ECG testing. Yet, 5 of the 18 subjects could not be paced above $120 \mathrm{bpm}$, and this limited the number of observations at higher HRs. Reaching the Wenkebach point at such low HRs was unexpected.

Final, STC-VM was analysed only for stepwise increases in $\mathrm{HR}$, and that there was no analysis for controlled experimental decrease in HR. This limitation in the protocol was necessary because of practical considerations for subjects and time on the table in the catheterization laboratory. The study design did not allow examination of the previously reported phenomenon of hysteresis (Viik et al., 1999).

In summary, STC-VM changes during paced HR increases were demonstrated in clinical subjects in the absence of ischemia. These STC-VM changes appeared to have a linear relation to HR increase of approximately $15-20 \mu \mathrm{V}$ per 10 beat 
increases for HRs above $100 \mathrm{~min}^{-1}$. The majority of subjects with HRs above $120 \mathrm{bpm}$ reached STC-VM levels above $50 \mu \mathrm{V}$. $\mathrm{J}$ point temporal relation to ST detection had no effect on the course of STC-VM during HR increases. The ST-VM was impossible to interpret during HR increases due to a large degree of variability in start levels and course. We conclude that STC-VM behaviour during HR increases at these levels, in the absence of ischemia, is systematic and predictable, and can be described by a relatively steep linear relationship. Any HR change-related adjustment of STC-VM levels needs to be prospectively tested for predictive value in detecting ischemia in both ischemic and non-ischemic patients. The time point at which the ST-deviation is measured is not as important at higher HRs as has been previously suggested, and has no effect on STCVM relation to HR at these HR levels. Further clinical testing is needed to improve the predictive value of STC-VM measurements during higher HRs, and particularly in relation to exercise and stress-ECG testing.

\section{Acknowledgments}

The authors would like to acknowledge the valuable assistance in data collection from Folke Rönn, M.D., Milos Kesek M.D. and Ulla-Britt Diamant, biomedical technician. This study was supported by grants from The Heart Foundation of Northern Sweden, Swedish Medical Research Council, Swedish HeartLung Fund, and Umeå University.

\section{References}

Alpert JS, Thygesen K, Antman E, Bassand JP. Myocardial infarction redefined-a consensus document of The Joint European Society of Cardiology/American College of Cardiology Committee for the redefinition of myocardial infarction. J Am Coll Cardiol (2000); 36: 959-969.

Bjurö T, Gullestad L, Endresen K, Nordlander M, Malm A, Höglund L, Wahlqvist I, Pernow J. Evaluation of ST-segment changes during and after maximal exercise tests in one-, two- and three-vessel coronary artery disease. Scand Cardiovasc J (2004); 38: 270-277.

Debbas NM, Jackson SH, de Jonghe D, Robert A, Camm AJ. Human atrial repolarization: effects of sinus rate, pacing and drugs on the surface electrocardiogram. J Am Coll Cardiol (1999); 33: 358-365.

Dellborg M, Topol EJ, Swedberg K. Dynamic QRS complex and ST segment vectorcardiographic monitoring can identify vessel patency in patients with acute myocardial infarction treated with reperfusion therapy. Am Heart J (1991); 122: 943-948.

Elamin MS, Boyle R, Kardash MM, Smith DR, Stoker JB, Whitaker W, Mary DA, Linden RJ. Accurate detection of coronary heart disease by new exercise test. Br Heart J (1982); 48: 311-320.

Frank E. Accurate, clinically practical system for spatial vectorcardiography. Circulation (1956); 13: 737-744.

Gibbons RJ, Balady GJ, Bricker JT, Chaitman BR, Fletcher GF, Froelicher VF, Mark DB, McCallister BD, Mooss AN, O’Reilly MG, Winters WL, Gibbons RJ, Antman EM, Alpert JS, Faxon DP, Fuster V, Gregoratos G, Hiratzka LF, Jacobs AK, Russell RO, Smith SC. ACC/AHA 2002 guideline update for exercise testing: summary article: a report of the
American College of Cardiology/American Heart Association Task Force on Practice Guidelines (Committee to Update the 1997 Exercise Testing Guidelines). Circulation (2002); 106: 1883-1892.

Häggmark S, Hohner P, Östman M, Friedman A, Diamond G, Lowenstein E, Reiz S. Comparison of Hemodynamic, Electrocardiographic, Mechanical, and Metabolic Indicators of Intraoperative Myocardial Ischemia in Vascular Surgical Patients with Coronary Artery Disease. Anesthesiology (1989); 70: 19-25.

Hayashi H, Okajima M, Yamada K. Atrial T(Ta) wave and atrial gradient in patients with A-V block. Am Heart J (1976); 91: 689-698.

Jensen SM, Häggmark S, Johansson G, Näslund U. On-line computerized vectorcardiography: influence of body position, heart rate, radiographic contrast fluid and myocardial ischemia. Cardiology (1997); 88: 576-584.

Jensen SM, Johansson G, Osterman G, Reiz S, Näslund U. On-line computerized vectorcardiography monitoring of myocardial ischemia during coronary angioplasty: comparison with 12-lead electrocardiography. Coron Artery Dis (1994); 5: 507-514.

Jensen SM, Karp K, Rask P, Näslund U. Assessment of myocardium at risk with computerized vectorcardiography and technetium-99m-sestamibi-single photon emission computed tomography during coronary angioplasty. Scand Cardiovasc J (2002); 36: 11-18.

Kligfield P, Ameisen O, Okin PM. Heart rate adjustment of ST segment depression for improved detection of coronary artery disease. Circulation (1989); 79: 245-255.

Lachterman B, Lehmann KG, Detrano R, Neutel J, Froelicher VF. Comparison of ST segment/heart rate index to standard ST criteria for analysis of exercise electrocardiogram. Circulation (1990); 82: 44-50.

Lehtinen R, Sievänen H, Viik J, Turjanmaa V, Niemelä K, Malmivuo J. Accurate detection of coronary artery disease by integrated analysis of the ST-segment depression/heart rate patterns during the exercise and recovery phases of the exercise electrocardiography test. Am J Cardiol (1996); 78: 1002-1006.

Mirvis DM, Wilson JL. Determination of the spatial and intensity properties of atrial repolarization potentials in the dog. J Electrocardiol (1986); 19: 99-107.

Näslund U, Häggmark S, Johansson G, Reiz S. Quantification of myocardium at risk and detection of reperfusion by dynamic vectorcardiographic ST segment monitoring in a pig occlusion-reperfusion model. Cardiovasc Res (1993); 27: 2170-2178.

Okin PM, Bergman G, Kligfield P. Effect of ST segment measurement point on performance of standard and heart rate-adjusted ST segment criteria for the identification of coronary artery disease. Circulation (1991); 84: 57-66.

Okin PM, Kligfield P. Computer-based implementation of the ST-segment/heart rate slope. Am J Cardiol (1989); 64: 926-930.

Pilhall M, Riha M, Jern S. Ischaemic heart disease and the changes in the QRS and ST segments during exercise: a pilot study with a novel vectorcardiographic system. Clin Physiol (1992); 12: 209-223.

Savvides M, Ahnve S, Bhargava V, Froelicher V. Computer analysis of exercise-induced changes in electrocardiographic variables. Comparison of methods and criteria. Chest (1983); 84: 699-706.

Sederholm M, Erhardt L, Sjögren A. Continuous vectorcardiography in acute myocardial infarction. Natural course of ST and QRS-vectors. Int J Cardiol (1983); 4: 53-63.

Viik J, Lehtinen R, Malmivuo J. Detection of coronary artery disease using maximum value of ST/HR hysteresis over different number of leads. J Electrocardiol (1999); 32(Suppl.): 70-75.

Wilson FN, Johnston FD. The vectorcardiogram. Am Heart J (1938); 16: $14-28$. 